\title{
A note on complete hyperbolic structures on ideal triangulated 3-manifolds
}

\author{
Feng Luo \\ Dedicated to Bus Jaco on the occasion of his 70th birthday
}

\begin{abstract}
It is a theorem of Casson and Rivin that the complete hyperbolic metric on a cusp end ideal triangulated 3-manifold maximizes volume in the space of all positive angle structures. We show that the conclusion still holds if some of the tetrahedra in the complete metric are flat.
\end{abstract}

\section{Introduction}

1.1. Epstein and Penner [1] proved that a non-compact finite volume complete hyperbolic 3-manifold has a geodesic triangulation in which each 3-simplex is a (possibly flat) ideal hyperbolic tetrahedron. Here a flat ideal hyperbolic tetrahedron is a tetrahedron with all dihedral angles being 0 and $\pi$. The purpose of this paper is to show that a geodesic ideal triangulation of a complete hyperbolic 3 -manifolds with some flat tetrahedra maximizes the volume in the closure of the space of non-negative angle structures. In the case all tetrahedra are non-flat, this was proved by Casson and Rivin (see 2], 3 for a written proof). We remark that the corresponding result also holds for hyper-ideal geodesic triangulations of compact hyperbolic 3-manifolds with totally geodesic boundary. This will be addressed in another paper.

1.2. Recall that a triangulated closed pseudo 3 -manifold $\left(M^{*}, T^{*}\right)$ is the quotient of a disjoint union of tetrahedra so that co-dimension- 1 faces are identified in pairs by affine homeomorphisms. The simplices in the triangulation $T^{*}$ are the quotients of simplices in the disjoint union. If we remove all vertices of $T^{*}$ from $M^{*}$, the result is an ideal triangulated non-compact 3-manifold $(M, \mathbf{T})$. We say $M$ has cusp ends if the link of each vertex in $T^{*}$ is a torus or a Kleinbottle. We will deal with ideal triangulated cusp end manifolds $(M, \mathbf{T})$ in this paper. An angle structure on $(M, \mathbf{T})$, introduced by Casson, Rivin and Lackenby $(\mathbf{7})$, assigns each edge of each tetrahedron a positive number, called the angle, so that

(1) the sum of three angles at edges from each vertex of each tetrahedron is $\pi$, and

1991 Mathematics Subject Classification. Primary 54C40, 14E20; Secondary 46E25, 20C20.

Key words and phrases. hyperbolic metric, 3-manifolds, tetrahedra, volume.

The work is supported in part by a NSF Grant. 
(2) the sum of angles around each edge is $2 \pi$.

An angled tetrahedron is a tetrahedron so that each edge has assigned a positive number called the angle so that condition (1) above holds. Given an angled tetrahedron, there is a unique ideal hyperbolic tetrahedron, call the geometric realization, whose dihedral angles are the assigned angles. The volume of an angled tetrahedron is defined to be the volume of its geometric realization. The volume of an angle structure is the sum of the volume of its angled tetrahedra.

For an ideal triangulated 3-manifold $(M, \mathbf{T})$ with $n$ tetrahedra, let $\mathbf{A}(\mathbf{T}) \subset \mathbf{R}^{6 n}$ be the space of all angle structures on $\mathbf{T}$ and let $v o l: \mathbf{A}(\mathbf{T}) \rightarrow \mathbf{R}$ be the volume function. By the Lobachevsky-Milnor's formula for volume, the volume function extends continuously to $\mathrm{vol}: \overline{\mathbf{A}(T)} \rightarrow \mathbf{R}$ where $\overline{\mathbf{A}(\mathbf{T})}$ is the (compact) closure of $\mathbf{A}(\mathbf{T})$ in $\mathbf{R}^{6 n}$.

Theorem 1.1 (Casson-Rivin). For an ideal triangulated cusped 3-manifold ( $M, \mathbf{T})$ so that $\mathbf{A}(\mathbf{T}) \neq \emptyset$, a point $p \in \mathbf{A}(\mathbf{T})$ corresponds to a complete hyperbolic structure on $M$ if and only if $p$ is the maximum point of the volume function vol : $\mathbf{A}(\mathbf{T}) \rightarrow \mathbf{R}$.

Since the space $\mathbf{A}(\mathbf{T})$ is non-compact, there is no guarantee that the maximum point of $\mathrm{vol}$ exists. Our main theorem generalizes theorem 1.1 in one direction.

Theorem 1.2. For an ideal triangulated cusp end 3-manifold $(M, \mathbf{T})$ so that $\overline{\mathbf{A}(\mathbf{T})} \neq$ $\emptyset$, if a point $p \in \overline{\mathbf{A}(\mathbf{T})}$ corresponds to a complete hyperbolic structure on $M$, then $p$ is the maximum point of the volume function vol $: \overline{\mathbf{A}(\mathbf{T})} \rightarrow \mathbf{R}$. Furthermore, the point $p$ is the unique maximum volume point in $\overline{\mathbf{A}(\mathbf{T})}$.

In [4], Futer and Gueritaud found an example of $(M, \mathbf{T})$ so that $\mathbf{A}(\mathbf{T}) \neq \emptyset$ and the maximum point of $\mathrm{vol}$ on $\overline{\mathbf{A}(\mathbf{T})}$ does not correspond to a complete hyperbolic metric. (A similar example was also found by Luo-Zheng [5] by making five 2-3 moves on the standard triangulation of the figure- 8 knot complement). This shows that theorem 1.2 cannot be improved to be a necessary and sufficient condition. We are curious if the uniqueness of the maximum volume on $\overline{\mathbf{A}(\mathbf{T})}$ is the sufficient condition for the completeness of the metric.

Theorem 1.2 will be proved in $\S 2$.

1.3. Acknowledgement. We thank D. Futer, F. Gueritaud and S. Tillmann for helpful conversations. The work is partially supported by the NSF.

\section{Proof of Theorem 1.2}

Let us begin with some notations and conventions. Let $\mathbf{R}_{>0}=\{x \in \mathbf{R} \mid x>0\}$ and $\mathbf{R}_{\geq 0}=\{x \in \mathbf{R} \mid x \geq 0\}$. Given a set $X$, let $\mathbf{R}^{X}=\{f: X \rightarrow \mathbf{R}\}$ be the vector space of all functions from $X$ to $\mathbf{R}$. The function $x \ln x:(0, \infty) \rightarrow \mathbf{R}$ is extended continuously to $[0, \infty) \rightarrow \mathbf{R}$ by setting $0 \ln 0=0$. Suppose $(M, \mathbf{T})$ is an ideal triangulated 3-manifold so that $V, E$ and $T$ are the sets of all (ideal) vertices, edges and tetrahedra. Let $I=\{(e, \sigma) \in E \times T \mid$ edge $e$ is adjacent to the tetrahedron $\sigma\}$. An angle structure is a vector in the space $\mathbf{R}^{I}$ satisfying a set of linear equations and linear inequalities. If $x \in \mathbf{R}^{I}$, we use $x_{i}$ to denote $x(i)$. If $i=(e, \sigma) \in I$, we use $i>e$ and $i<\sigma$ to indicate the incident relation. Three distinct $i=\left(e_{1}, \sigma\right), j=\left(e_{2}, \sigma\right)$ and $k=\left(e_{3}, \sigma\right)$ in $I$ so that $e_{1}, e_{2}, e_{3}$ are three edges from the same vertex in $\sigma$ will be denoted by $\{i, j, k\} \in \Delta$. Finally, we use $(e, \sigma) \sim\left(e^{\prime}, \sigma\right)$ to indicate that $e, e^{\prime}$ are two opposite edges in the same tetrahedron $\sigma$. Using these notations, we have 


$$
A(\mathbf{T})=\left\{x \in \mathbf{R}^{I} \mid(1),(2),(3) \text { hold }\right\}
$$

where

(1) if $\{i, j, k\} \in \Delta, x_{i}+x_{j}+x_{k}=\pi$;

(2) if $e \in E, \sum_{i \in I, i>e} x_{i}=2 \pi$;

(3) $x_{i}>0$ for all $i \in I$.

Note that condition (1) implies that $x_{i}=x_{j}$ when $i \sim j$. The closure $\overline{\mathbf{A}(\mathbf{T})}$ of $\mathbf{A}(\mathbf{T})$ is give by $\left\{x \in \mathbf{R}_{\geq 0}^{I} \mid\right.$ (1) and (2) hold $\}$. Theorem 1.2 does not assume $\mathbf{A}(\mathbf{T}) \neq \emptyset$, but it assumes $\overline{\mathbf{A}(\mathbf{T})} \neq \emptyset$. Elements in $\overline{\mathbf{A}(\mathbf{T})}$ will be called non-negative angle structures.

Suppose $\sigma$ is an angled tetrahedron with three angles $x_{1}, x_{2}, x_{3}$ at three edges from a vertex. Then the Lobachevsky-Milnor volume formula says the volume $\operatorname{vol}(\sigma)$ of $\sigma$ is $\Lambda\left(x_{1}\right)+\Lambda\left(x_{2}\right)+\Lambda\left(x_{3}\right)$ where $\Lambda(t)=-\int_{0}^{t} \ln |2 \sin (u)| d u$ is the Lobachevsky function. The function $\Lambda(t)$ is continuous on $[0, \pi]$. In particular, the volume function $v o l: \overline{\mathbf{A}(\mathbf{T})} \rightarrow \mathbf{R}$ is give by

$$
\operatorname{vol}(x)=\frac{1}{2} \sum_{i \in I} \Lambda\left(x_{i}\right)
$$

Note that $\operatorname{vol}(x)=\sum_{\sigma \in T} \operatorname{vol}(\sigma)$ where $\sigma$ is the angled tetrahedron with angles given by $x$.

2.1. Set up the proof. Assume that $p \in \overline{\mathbf{A}(\mathbf{T})}$ corresponds to the complete hyperbolic metric on $M$. If $p \in \mathbf{A}(\mathbf{T})$, then Casson-Rivin's theorem implies that $p$ is the maximum point of the volume. It remains to deal with $p \in \partial \mathbf{A}(\mathbf{T})=\overline{\mathbf{A}(\mathbf{T})}-$ $\mathbf{A}(\mathbf{T})$. Take $q \in \overline{\mathbf{A}(\mathbf{T})}$ so that $q \neq p$. The goal is to show that $\operatorname{vol}(p)>\operatorname{vol}(q)$. To this end, let $f(t)=\operatorname{vol}((1-t) p+t q)$ for $t \in[0,1]$. We will show that $f(0)>f(1)$, i.e., $\operatorname{vol}(p)>\operatorname{vol}(q)$.

Lemma 2.1. The function $f(t)$ is concave in $[0,1]$ and is strictly concave in the open interval $(0,1)$.

Proof. By a result of Rivin [9], the volume function $\Lambda\left(t_{1}\right)+\Lambda\left(t_{2}\right)+\Lambda\left(t_{3}\right)$ is strictly concave in the set $\left\{\left(t_{1}, t_{2}, t_{3}\right) \in \mathbf{R}^{3} \mid t_{1}+t_{2}+t_{3}=\pi, t_{i}>0\right.$ for $\left.i=1,2,3\right\}$. In particular, this implies that the function $\operatorname{vol}(x)$ is concave in $x \in \overline{\mathbf{A}(\mathbf{T})}$. Thus $f(t)$ is concave in $[0,1]$. To see the strictly concavity, note that $\operatorname{vol}(p)>0$ since it is the volume of a complete hyperbolic structure. In particular, there is tetrahedron $\sigma \in T$ so that its angles in $p$ are all positive. This implies that for $t \in(0,1)$, the angles of $\sigma$ in $(1-t) p+t q$ are positive. By Rivin's theorem, the volume $\sum_{i \in I, i<\sigma} \Lambda\left((1-t) p_{i}+t q_{i}\right)$ is strictly concave in $t \in(0,1)$. Since $f(t)$ is the sum of concave functions in $t$ so that one of then is strictly concave, it follows that $f(t)$ is strictly concave in $(0,1)$.

For $t \in(0,1)$, by the definition of the volume (2.1), we have

$$
f^{\prime}(t)=-\frac{1}{2} \sum_{i \in I}\left(q_{i}-p_{i}\right) \ln \left|2 \sin \left((1-t) p_{i}+t q_{i}\right)\right|
$$

Note that we have used the convention that $0 \ln 0=0$ in (2.2). Indeed, if $p_{i}=q_{i}$ is 0 or $\pi$, then the term in (2.2) corresponding to $i$ is defined to be 0 . (This is due to the fact that $\Lambda(x)+\Lambda(y)+\Lambda(\pi-x-y)=0$ if $x \in \pi \mathbf{Z}$. ) 
The goal is to show that

$$
\lim _{t \rightarrow 0^{+}} f^{\prime}(t) \leq 0 .
$$

Note that (2.3) and lemma 2.1 imply that $f(0)>f(1)$. In the rest of the subsections, we will focus on proving (2.3).

2.2. Let $J=\left\{i \in I \mid p_{i}=0\right.$ or $\left.p_{i}=\pi\right\}$. Note that if $(e, \sigma) \in J$, then $\left(e^{\prime}, \sigma\right) \in J$ for all other edges $e^{\prime}$ in $\sigma$ by the definition of flat tetrahedron, i.e., all its dihedral angles are 0 or $\pi$. Let $a=q-p \in \mathbf{R}^{I}$.

Lemma 2.2. (1) If $\{i, j, k\} \in \Delta$, then $a_{i}+a_{j}+a_{k}=0$, i.e., for each tetrahedron $\sigma, \frac{1}{2} \sum_{i<\sigma} a_{i}=0$.

(2) For each edge $e \in E, \sum_{i>e} a_{i}=0$.

(3) $\sum_{i \in I} a_{i}=0$.

Indeed, the first two conditions follows from the definition of angle structures (1) and (2). The last condition follows from part (1) by summing over all tetrahedra and then divided by 2 .

By lemma 2.2(3), we can rewrite $f^{\prime}(t)$ in $(2.2)$ as

$$
f^{\prime}(t)=-\frac{1}{2} \sum_{i \in I} a_{i} \ln \left|\sin \left((1-t) p_{i}+t q_{i}\right)\right|
$$

The following was proved in [6]. It can also be found in [4].

\section{Lemma 2.3.}

$$
\lim _{t \rightarrow 0^{+}} f^{\prime}(t)=-\frac{1}{2}\left(\sum_{i \notin J} a_{i} \ln \left|\sin \left(p_{i}\right)\right|-\sum_{i \in J} a_{i} \ln \left|a_{i}\right|\right) .
$$

2.3. Penner's decorated ideal simplexes. To understand the right-handside of (2.5), we need a proposition about the geometry of decorated ideal hyperbolic tetrahedra. Following Penner [8, a decorated ideal n-simplex is an ideal hyperbolic $\mathrm{n}$-simplex so that each vertex is assigned a horosphere centered at the vertex. If $\sigma$ is a decorated ideal n-simplex and $e$ is an edge of it, the length $L(e)$ of $e$ is defined to be the signed distance between the two horospheres centered at the end points of $e$ (the distance is negative if the horospheres intersect). More precisely, suppose $p, p^{\prime}$ are the two points of intersection of $e$ with these two horocspheres. Then $L(e)$ is $\operatorname{dist}\left(p, p^{\prime}\right)$ if these two horospheres are disjoint and is $-\operatorname{dist}\left(p, p^{\prime}\right)$ if they intersect.

Lemma 2.4. Suppose $\sigma$ is a decorated ideal hyperbolic tetrahedron with edge length $L(e)$ and dihedral angle $\theta(e)$ at the edge $e$. Assume that $\theta(e) \in(0, \pi)$ for all edges. Then there is a constant $c(\sigma)$ depending only on $\sigma$ so that for any pairs of opposite edges $e, e^{\prime}$ in $\sigma$,

$$
\frac{1}{2}\left(L(e)+L\left(e^{\prime}\right)\right)=\ln |\sin (\theta(e))|+c(\sigma)
$$

Proof. The proof is based on the cosine law for decorated ideal triangles first discovered by Penner $\left[\mathbf{8}\right.$. Namely, give a decorated ideal triangle of lengths $l_{1}, l_{2}, l_{3}$, the "angles" of the triangle, denoted by $a_{1}, a_{2}, a_{3}$, are the lengths of the portion of the horocycle inside the triangle. Indices are arranged so that the angle $a_{i}$ is facing the edge of length $l_{i}$. The the cosine law says

$$
l_{i}=-\left(\ln a_{j}+\ln a_{k}\right) \quad\{i, j, k\}=\{1,2,3\} .
$$


For the edge $e$ (respectively $e^{\prime}$ ), there are two face triangles of the tetrahedron $\sigma$ having $e$ (resp. $e^{\prime}$ ) as an edge. These face triangles are naturally decorated ideal hyperbolic triangles. Let $a_{1}, a_{2}, a_{3}, a_{4}$ (resp. $a_{1}^{\prime}, a_{2}^{\prime}, a_{3}^{\prime}, a_{4}^{\prime}$ ) be the inner angles of these decorated face ideal triangles so that $a_{i}$ 's are adjacent to $e$ (resp. $e^{\prime}$ ). Let the rest of the four face angles (of the four decorated ideal triangles) be $b_{1}, \ldots, b_{4}$. Here vertices of angles $b_{i}$ are either in $e$ or $e^{\prime}$. Then by the cosine law, we have

$$
L(e)=-\frac{1}{2} \sum_{i=1}^{4} \ln a_{i} \quad \text { and } \quad L\left(e^{\prime}\right)=-\frac{1}{2} \sum_{i=1}^{4} \ln a_{i}^{\prime}
$$

This shows

$$
\begin{gathered}
L(e)+L\left(e^{\prime}\right)=-\frac{1}{2} \sum_{i=1}^{4}\left(\ln a_{i}+\ln a_{i}^{\prime}\right) \\
=\frac{1}{2} c_{0}(\sigma)+\frac{1}{2} \sum_{i=1}^{4} \ln b_{i}
\end{gathered}
$$

where $c_{0}(\sigma)=\sum_{i=1}^{4}\left(\ln a_{i}+\ln a_{i}^{\prime}+\ln b_{i}\right)$ is the sum over all twelve face angles.

Consider the Euclidean triangles obtained by intersecting the horospheres with the ideal tetrahedron. The dihedral angles $\theta(e)$ 's are the inner angles and the face angles $b_{i}$ 's are the edge lengths of the Euclidean triangles. Thus, by the Sine law for Euclidean triangles, we can write

$$
\ln b_{i}=c_{i}(\sigma)+\ln \mid \sin (\theta(e) \mid
$$

where $b_{i}$ has its vertex at $e$. Putting these together and using the fact that $\theta(e)=$ $\theta\left(e^{\prime}\right)$, we obtain $(2.6)$ where $c(\sigma)=\frac{1}{2} \sum_{i=0}^{4} c_{i}(\sigma)$.

For a decorated ideal triangle of edge lengths $L(e)$, we define the average edge length of $e$ to be $W(e)=\frac{1}{2}\left(L(e)+L\left(e^{\prime}\right)\right)$ where $e, e^{\prime}$ are opposite edges.

Lemma 2.5. For a decorated ideal tetrahedron $\sigma$, if $e_{1}, e_{2}, e_{3}$ are three edges from a vertex $v$, then

$$
e^{W\left(e_{1}\right)}+e^{W\left(e_{2}\right)} \geq e^{W\left(e_{3}\right)}
$$

so that equality holds if and only if $\theta\left(e_{3}\right)=\pi, \theta\left(e_{1}\right)=\theta\left(e_{2}\right)=0$.

Indeed, consider the Euclidean triangle obtained by intersecting the horosphere centered at the vertex $v$ with the ideal tetrahedron. The inner angles of the Euclidean triangle are $\theta\left(e_{i}\right)$ 's and the edge lengths of it are $R \sin \left(\theta\left(e_{i}\right)\right)$ where $R$ is the radius of the circumcircle. Now by lemma 2.4 that $\sin \left(\theta\left(e_{i}\right)\right)=c^{\prime} e^{W\left(e_{i}\right)}$, the lengths of the edges in the triangle are $c e^{W\left(e_{i}\right)}$ for some constant $c$. Thus the lemma follows from the triangular inequality for edge lengths of triangles.

2.4. A proof of theorem 1.2. Recall that the maximum volume point $p \in$ $\overline{\mathbf{A}(\mathbf{T})}$ corresponds to a complete hyperbolic metric, i.e., there exists a geodesic triangulation of a complete hyperbolic metric on $M$ so that the triangulation is isotopic to $T$ and the dihedral angles coincide with the angles given by $p$. Choose small horospheres at the cusp ends of $M$ so that each tetrahedron becomes an ideal decorated hyperbolic tetrahedron. In particular, each edge $e$ in $\mathbf{T}$ has a the edge 
length $L(e)$ (in the decorated tetrahedra). For each $i=(e, \sigma) \in I$, we define the average length $w_{i}$ (of $e$ in $\sigma$ ) to be

$$
w_{i}=\frac{1}{2}\left(L(e)+L\left(e^{\prime}\right)\right)
$$

where $e, e^{\prime}$ are opposite edges in $\sigma$.

Lemma 2.6. We have

$$
\sum_{i \notin J} a_{i} \ln \left|\sin \left(p_{i}\right)\right|=\sum_{i \notin J} a_{i} w_{i} .
$$

Proof. A tetrahedron $\sigma$ is called flat (in $p$ ) if its dihedral angles in $p$ are $0,0, \pi$, i.e., there is $i \in I$ with $i<\sigma$. If $\sigma$ is not flat, then by lemma 2.4, there is a constant $c(\sigma)$ so that (2.6) holds for each pair of opposite edges $e, e^{\prime}$. This is the same as

$$
w_{i}=\ln \left|\sin \left(p_{i}\right)\right|+c(\sigma)
$$

for $i<\sigma$. Multiply (2.12) by $a_{i}$ and sum over all not flat tetrahedra, we obtain

$$
\sum_{i \notin J} a_{i} w_{i}=\sum_{i \notin J} a_{i} \ln \left|\sin \left(p_{i}\right)\right|+\sum_{i \notin J, i<\sigma} a_{i} c(\sigma) .
$$

But

$$
\sum_{i \notin J, i<\sigma} a_{i} c(\sigma)=\sum_{\sigma \text { not flat }} c(\sigma)\left(\sum_{i<\sigma} a_{i}\right)=0
$$

due to lemma 2.2 (1). This ends the proof.

On the other hand, we have

$$
\sum_{i \in I} a_{i} w_{i}=0
$$

Indeed, if $i=(e, \sigma)$ and $j=\left(e^{\prime}, \sigma\right)$ where $e, e^{\prime}$ are opposite edges in $\sigma$, then $a_{i}=a_{j}$ and $w_{i}=w_{j}$. Furthermore by (2.10), $a_{i} w_{i}+a_{j} w_{j}=a_{i} L(e)+a_{j} L\left(e^{\prime}\right)$. Thus

$$
\begin{gathered}
\sum_{i \in I} a_{i} w_{i}=\sum_{i \in I} a_{i}\left(\sum_{e<i} L(e)\right) \\
=\sum_{e \in E} L(e)\left(\sum_{i<e} a_{i}\right) \\
=0
\end{gathered}
$$

due to lemma $2.2(2)$.

By combining (2.11) and (2.14), we obtain

$$
\sum_{i \notin J} a_{i} \ln \left|\sin \left(p_{i}\right)\right|=-\sum_{i \in J} a_{i} w_{i} .
$$

By (2.15), we can rewrite (2.5) as,

$$
\begin{gathered}
\lim _{t \rightarrow 0^{+}} f^{\prime}(t)=-\frac{1}{2}\left(\sum_{i \in J} a_{i} \ln \left|a_{i}\right|+\sum_{i \in J} a_{i} w_{i}\right) \\
=\sum_{\sigma \text { is flat }}\left(-a_{i} \ln \left|a_{i}\right|-a_{j} \ln \left|a_{j}\right|-a_{k} \ln \left|a_{k}\right|+a_{i} w_{i}+a_{j} w_{j}+a_{k} w_{k}\right)
\end{gathered}
$$

where $i, j, k<\sigma,\{i, j, k\} \in \Delta$. 
Since $\sigma$ is flat, we may assume that $p_{k}=\pi, p_{i}=p_{j}=0$. Then by lemma 2.5, three average lengths $w_{i}, w_{j}, w_{k}$ satisfy the triangular equality, i.e., $e^{w_{k}}=e^{w_{i}}+e^{w_{j}}$. Furthermore, $a_{i} \geq 0, a_{j} \geq 0$ and $a_{i}+a_{j}+a_{k}=0$.

We claim

$$
-a_{i} \ln \left|a_{i}\right|-a_{j} \ln \left|a_{j}\right|-a_{k} \ln \left|a_{k}\right|+a_{i} w_{i}+a_{j} w_{j}+a_{k} w_{k} \leq 0 .
$$

Evidently, (2.16) implies that $\lim _{t \rightarrow 0^{+}} f^{\prime}(t) \leq 0$. Now (2.16) follows from the following simple lemma on a convex function where we take $x=a_{i}, y=a_{j}, z=$ $a_{k}, a=w_{i}, b=w_{j}, c=w_{k}$.

Lemma 2.7. Suppose $x, y, a, b, c \in \mathbf{R}_{\geq 0}, x+y+z=0$ and $e^{c} \geq e^{a}+e^{b}$. Then

$$
-x \ln x-y \ln y-z \ln z+a x+b y+c z \leq 0 .
$$

We remark that if $e^{c}=e^{a}+e^{b}$, then the inequality becomes equality for some non-zero $x, y, z$.

Proof. Replacing $z=-x-y$, we obtain the equivalent form of (2.17) as

$$
(x+y) \ln (x+y)-x \ln x-y \ln y \leq(c-a) x+(c-b) y .
$$

The above inequality is homogeneous in $(x, y)$, i.e., it is equivalent if we replace $(x, y)$ by $(\lambda x, \lambda y)$ where $\lambda>0$. Thus we may assume further that $y=1$. Thus it remains to prove,

$$
(x+1) \ln (x+1)-x \ln x \leq(c-a) x+(c-b)
$$

for all $x \geq 0$. Let $g(x)=(x+1) \ln (x+1)-x \ln x$. Then $g^{\prime}(x)=\ln \left(1+\frac{1}{x}\right)$ and $g^{\prime \prime}(x)=-\frac{1}{x(1+x)}$. It follows that $g^{\prime \prime}(x) \leq 0$, i.e., $g$ is concave in $[0, \infty)$. The equation of the tangent line to $g$ at the point $x_{0}=\frac{e^{a}}{e^{c}-e^{a}}$ is $y=h(x)$ where

$$
h(x)=(c-a) x+\left(c-\ln \left(e^{c}-e^{a}\right)\right) .
$$

Since $g$ is concave, we have $g(x) \leq h(x)$. Now use $b \geq \ln \left(e^{c}-e^{a}\right)$, we obtain $h(x) \leq(c-a) x+(c-b)$. Thus lemma follows.

\section{References}

[1] Epstein, D. B. A.; Penner, R. C. Euclidean decompositions of noncompact hyperbolic manifolds. J. Differential Geom. 27 (1988), no. 1, 67-80.

[2] Futer, D., F. Gueritaud, F; From angled triangulations to hyperbolic structures, arXiv:1004.0440 to appear in the proceedings of the Columbia University workshop on interactions between hyperbolic geometry, quantum topology, and number theory.

[3] Chan, ken;, Constructing hyperbolic 3-manifolds, Undergraduate thesis with Craig Hodgson, University of Melbourne, 2002.

[4] Guritaud, Franois On canonical triangulations of once-punctured torus bundles and two-bridge link complements. With an appendix by David Futer. Geom. Topol. 10 (2006), 1239-1284.

[5] Luo, F., H. Zheng, maximum volume angle structures on figure-8 complement, unpublished note, 2006

[6] Luo, F., Volume optimization, normal surfaces and Thurstons equation on triangulated 3manifolds, arXiv:0903.1138

[7] Lackenby, Marc; Word hyperbolic Dehn surgery. Invent. Math. 140 (2000), no. 2, 243-282.

[8] Penner, R. C.; The decorated Teichmller space of punctured surfaces. Comm. Math. Phys. 113 (1987), no. 2, 299-339.

[9] Rivin, Igor; Euclidean structures on simplicial surfaces and hyperbolic volume. Ann. of Math.

(2) 139 (1994), no. 3, 553-580.

Department of Mathematics, Rutgers University, New Brunswick, New Jersey 08854 E-mail address: fluo@math.rutgers.edu 